\title{
Monogenic Obesity
}

National Cancer Institute

\section{Source}

National Cancer Institute. Monogenic Obesity. NCI Thesaurus. Code C120382.

Obesity associated with an identifiable mutation in a single gene. 